\title{
Das infizierte Ellenbogengelenk: Diagnostik und Therapie
}

\author{
Andreas Heinrich Tiemann, Rainer Braunschweig, Gunther Olaf Hofmann
}

\section{Zusammenfassung}

Septische Arthritiden des Ellenbogens sind sehr selten. Insofern stellen sie eine diagnostische und therapeutische Herausforderung dar. In diesem Manuskript werden die modernen diagnostischen und therapeutischen Verfahren für diese Entität dargestellt. Die Diagnostik basiert einerseits auf der Erhebung einer präzisen Anamnese und der anschließenden klinischen Untersuchung. Andererseits kommen die entsprechenden Laboruntersuchungen, mikrobiologische und histologische Analysen und die breite Palette bildgebender Verfahren zur Anwendung. Die Therapie umfasst nonoperative und operative Verfahren. Die nonoperativen Verfahren können, im Frühstadium der Erkrankung angewandt, zu einem Symptomrückgang führen. Im Spätstadium ist die chirurgische Revision zwingend notwendig. Nicht immer ist eine Restitutio ad integrum möglich. Neben der Implantation einer Gelenkendoprothese stellen Arthrodese, Resektionsarthroplastik oder schlimmstenfalls die Amputation mögliche Alternativen dar.

\section{The Infected Elbow: Diagnostics and Therapy}

Due to the fact that septic elbow arthritis is a very rare disease it represents a difficult diagnostic and therapeutic challenge. The purpose of the present contibution is to provide an update on the diagnostic features of and the therapy for septic elbow arthritis. Modern diagnostic methods are still based on the patient's individual history and the local clinical changes. On the other hand, they nowadays include laboratory tests, microbiological and histological examination and all forms of imaging procedures. The therapy includes operative surgical revision and non-operative methods like the administration of antibiotics. The non-operative management may lead to the relief of symptoms in the early stages of septic elbow arthritis. In the later stages surgical revision including synovectomy may be undertaken in order to preserve the joint. Restitutio ad integrum is not always achievable. Next to arthroplasty arthrodesis, resection arthroplasty or in extreme case amputation may be needed.

\section{Definition}

Bei der infektiösen Arthritis des Ellenbogens handelt es sich um eine erregerinduzierte Infektion des Gelenks. Als Erreger kommen Bakterien, Viren, Pilze und auch Parasiten in Betracht. Je nach Ausdehnung der Erkrankung (zeitlich und räumlich) werden neben der Synovia zunächst sämtliche unmittelbar dem Gelenk zuzuordnenden anatomischen Strukturen (sog. innere Gelenkanteile),

OP-JOURNAL 2012; 28: 82-91

(c) Georg Thieme Verlag KG Stuttgart · New York DOI http://dx.doi.org/10.1055/s-0031-1298484

dann die angrenzenden Knochen und Weichteile in die Infektion mit einbezogen [49].

Synonym für den Terminus der infektiösen Arthritis werden folgende Begriffe benutzt: septische, eitrige Arthritis, Pyarthros, Gelenkinfektion.

\section{Klassifikation}

In Analogie zu den Klassifikationen septischer Arthritiden an anderer Lokalisation können Infektionen des Ellenbogengelenks wie folgt unterteilt werden:

- nach dem Infektionsweg

- der Akuität
- nach dem auslösenden Erreger

- nach dem pathologisch/anatomischen Erscheinungsbild

- nach dem klinischen Bild

- nach dem intraoperativen/radiologischen klinischen Befund

Betrachtet man die klinische Relevanz der einzelnen Klassifikationen, so treten die folgenden in den Vordergrund:

\section{a. Einteilung nach dem Infektionsweg}

Primäre/exogene Gelenkinfektion. Die Erreger dringen von außen in das Gelenk ein (z.B. posttraumatisch, postoperativ oder nach diagnostischen Maßnahmen).

Sekundäre/endogene Gelenkinfektion. Die Erreger gelangen durch eine endogene Streuung über die Blutbahn in die Gelenke.

\section{b. Einteilung nach der Akuität}

Frühinfekt

Der Zeitraum zwischen dem Auftreten von Symptomen und dem ursächlichen Ereignis ist kleiner als 6 Wochen. Entscheidend ist hier das Kriterium der Reversibilität, welches in diesem Zeitraum noch gegeben ist. Grundsätzlich kann die konsequent durchgeführte Therapie zu einer Restitutio ad integrum der noch auf die Membrana synovialis beschränkten Erkrankung führen [18].

\section{Spätinfekt}

Der Zeitraum ist größer als 6 Wochen. In diesem Stadium hat die Erkrankung auf die Membrana fibrosa übergegriffen. Eine Restitutio ad integrum ist nicht mehr möglich [18].

Schmidt et al. unterscheiden den akuten vom chronischen Infekt. Als Grenze für den Übergang von der akuten zur chronischen Infektion legten sie einen Zeitraum von 7 Tagen fest [45]. 
Tab. 1 Stadiengerechtes Vorgehen beim Gelenkinfekt $[11,12,46]$.

\begin{tabular}{|c|c|c|c|c|c|c|c|}
\hline \multirow[t]{2}{*}{ Stadium nach Gächter } & \multicolumn{3}{|l|}{ Vorbehandlung } & \multicolumn{4}{|c|}{ Infektausdehnung } \\
\hline & V1 = keine & V2 = ASK & $\begin{array}{l}\text { V3 = offene } \\
\text { Revision }\end{array}$ & A Weichteile & B nur Gelenk & $\begin{array}{l}\text { C gelenknaher } \\
\text { Knochen }\end{array}$ & $\begin{array}{l}\text { D Gelenk + } \\
\text { gel. Knochen }\end{array}$ \\
\hline Stadium 1 & ASK Splg. & $1 \times$ ASK & offene Revision & ASK Splg. & ASK Splg. & offene Revision & offene Revision \\
\hline trüber Erguss & 10-20 I Spl. & $\begin{array}{l}\text { ggf. sofort } \\
\text { umsteigen }\end{array}$ & 10-20 I Spl. & 10-20 I Spl. & 10-20 I Spl. & 10-20 I Spl. & 10-20 I Spl. \\
\hline infiziertes Hämarthros & Antibiotikum & & $\begin{array}{l}14 \text { Tage Anti- } \\
\text { biotikum }\end{array}$ & Antibiotikum & Antibiotikum & $\begin{array}{l}14 \text { Tage Anti- } \\
\text { biotikum }\end{array}$ & $\begin{array}{l}14 \text { Tage Anti- } \\
\text { biotikum }\end{array}$ \\
\hline Synoviarötung & 5 Tage & & & 5 Tage & 5 Tage & & \\
\hline \multicolumn{8}{|l|}{ petechiale Einblutungen } \\
\hline Stadium 2 & ASK Splg. & $1 \times$ ASK & offene Revision & ASK Splg. & ASK Splg. & offene Revision & offene Revision \\
\hline Synovialitis & 10-20 I Spl. & $\begin{array}{l}\text { ggf. sofort } \\
\text { umsteigen }\end{array}$ & 10-20 I Spl. & 10-20 I Spl. & 10-20 I Spl. & 10-20 I Spl. & 10-20 I Spl. \\
\hline Fibrinbeläge & Antibiotikum & & $\begin{array}{l}14 \text { Tage Anti- } \\
\text { biotikum }\end{array}$ & Antibiotikum & Antibiotikum & $\begin{array}{l}14 \text { Tage Anti- } \\
\text { biotikum }\end{array}$ & $\begin{array}{l}14 \text { Tage Anti- } \\
\text { biotikum }\end{array}$ \\
\hline Eiter & 7 Tage & & & 7 Tage & 7 Tage & & \\
\hline Stadium 3 & offene Revision & offene Revision & offene Revision & offene Revision & offene Revision & offene Revision & offene Revision \\
\hline Zottenbildung & 10-20 I Spl. & 10-20 I Spl. & 10-20 I Spl. & 10-20 I Spl. & 10-20 I Spl. & 10-20 I Spl. & 10-20 I Spl. \\
\hline $\begin{array}{l}\text { hypertrophe } \\
\text { Synovialitis }\end{array}$ & $\begin{array}{l}14 \text { Tage Anti- } \\
\text { biotikum }\end{array}$ & $\begin{array}{l}14 \text { Tage Anti- } \\
\text { biotikum }\end{array}$ & $\begin{array}{l}14 \text { Tage Anti- } \\
\text { biotikum }\end{array}$ & $\begin{array}{l}14 \text { Tage Anti- } \\
\text { biotikum }\end{array}$ & $\begin{array}{l}14 \text { Tage Anti- } \\
\text { biotikum }\end{array}$ & $\begin{array}{l}14 \text { Tage Anti- } \\
\text { biotikum }\end{array}$ & $\begin{array}{l}14 \text { Tage Anti- } \\
\text { biotikum }\end{array}$ \\
\hline \multicolumn{8}{|l|}{ Knorpel angegriffen } \\
\hline \multicolumn{8}{|l|}{ „Synovialisschwamm“ } \\
\hline Stadium 4 & offene Revision & offene Revision & offene Revision & offene Revision & offene Revision & offene Revision & offene Revision \\
\hline $\begin{array}{l}\text { radiolog. Gelenk- } \\
\text { destruktion }\end{array}$ & 10-20 I Spl. & 10-20 I Spl. & 10-20 I Spl. & 10-20 I Spl. & 10-20 I Spl. & 10-20 I Spl. & 10-20 I Spl. \\
\hline "Synovialismalignität“ & $\begin{array}{l}14 \text { Tage Anti- } \\
\text { biotikum }\end{array}$ & $\begin{array}{l}14 \text { Tage Anti- } \\
\text { biotikum }\end{array}$ & $\begin{array}{l}14 \text { Tage Anti- } \\
\text { biotikum }\end{array}$ & $\begin{array}{l}14 \text { Tage Anti- } \\
\text { biotikum }\end{array}$ & $\begin{array}{l}14 \text { Tage Anti- } \\
\text { biotikum }\end{array}$ & $\begin{array}{l}14 \text { Tage Anti- } \\
\text { biotikum }\end{array}$ & $\begin{array}{l}14 \text { Tage Anti- } \\
\text { biotikum }\end{array}$ \\
\hline
\end{tabular}

\section{c. Einteilung nach dem auslösenden Erreger}

Battmann und Haas unterschieden 2004 virale und bakterielle Arthritiden von sog. Sonderformen (z.B. Gonokokkenarthritis, granulomatöse Arthritis, Pilzarthritis) [5]. Synonym können in diesem Zusammenhang die Begriffe spezifische und unspezifische Gelenkinfektion verwendet werden.

Den klassischen Erreger der infektiösen Arthritis des Ellenbogens gibt es genauso wenig wie den klassischen Erreger infektiöser Arthritiden in altero loco. Betrachtet man die einschlägige Literatur, so zeigt sich, dass S. aureus das Gros der Gelenkinfektionen der oberen Extremität verursacht $[9,35,37]$. In der Literatur finden sich dazu Angaben zwischen 41 und $87 \%[8,9,15,37,43]$. Häufig sind auch gramnegative Erreger zu identifizieren.
Multiresistente Erreger septischer Arthritiden treten in den letzten Jahren zunehmend in den Fokus [9]. Studien aus England zeigen einen Anteil von 6-8\% MRSA an den analysierten septischen Arthritiden $[8,15]$. Bereits 2005 wiesen Ross und Davidson bei 59 Fällen akuter Gelenkinfektionen in 25\% MRSA nach [3].

\section{d. Einteilung nach dem intraoperativen/ (radiologischen) Befund}

Hier hat sich die Einteilung nach Gächter durchgesetzt, die dem Gelenkinfekt in Abhängigkeit von seiner Ausprägung 4 Stadien zuordnet $[11,12]$. Modifiziert wurde diese Klassifikation durch Jensen und Schmidt [19,46] (Tab. 1). Diese Einteilung ist von großem Nutzen in der klinischen Praxis, da sich aus ihr Handlungsempfehlungen für die Therapie ableiten lassen.
Die Zusammenschau dieser 4 Klassifikationen führt zu konkreten Empfehlungen für das therapeutische Vorgehen, und zwar sowohl auf der operativen als auch auf der adjuvanten Seite.

\section{Inzidenz allgemein}

Insgesamt gesehen sind Gelenkinfektionen selten. Grundsätzlich können alle Gelenke befallen sein. Die Erhebung von Angaben zur Epidemiologie und Inzidenz septischer Arthritiden ist schwierig und wird durch verschiedene Faktoren limitiert [35]:

- Die Erhebung der Daten geschieht retrospektiv.

- Die Infektion gilt nur dann als gesichert, wenn ein bakteriologischer Keimnachweis gelingt.

Man geht heute davon aus, dass die Inzidenz septischer Arthritiden in Westeuropa zwischen 4 und 10 Fälle/100000 Patientenjahre/Jahr liegt [13,23,39]. 


\section{Inzidenz der Infektionen der oberen Extremität/Ellenbogengelenk}

Infektionen der großen Gelenke der oberen Extremität, insbesondere isolierte Infektionen des Ellenbogengelenks sind selten. Die Häufigkeit derartiger Infektionen liegt insgesamt bspw. deutlich unter der des Knie- oder Schultergelenks [21]. Allerdings befassen sich nur wenige Autoren mit dieser Entität. In ihrem Buch von 2002 berichten Josten und Lill über Zahlen der Mayo-Klinik, aus denen hervorgeht, dass von allen Infektionen der großen Gelenke des Erwachsenen nur ca. 6\% das Ellenbogengelenk betreffen [21]. Ältere Arbeiten aus den 60er- und 70er-Jahren gehen von einer Inzidenz von $9-13 \%$ aus $[2,14]$. Es finden sich weiterhin Berichte mit geringen Fallzahlen oder Einzelfallbeschreibungen [37]. In ihrer Untersuchung von 2006 beschreiben Metha et al. 52 Fälle von Gelenkinfektionen der oberen Extremität in einem Zeitraum von 10 Jahren. In 23 Fällen (44\%) handelte es sich dabei um isolierte Infektionen des Ellenbogens [37].

\section{Diagnostik}

Auch für das infizierte Ellenbogengelenk gilt die Regel, nach der die frühestmögliche Diagnose anzustreben ist. Nur so besteht eine Chance zum Erhalt des Gelenks.

Wie Mathews bereits 2008 ausführte, beeinflusst das Ausmaß der klinischen Erfahrung des behandelnden Arztes die Detektion und korrekte Diagnose eines Gelenkinfekts im Sinne eines „GoldStandards“ [34]. Jerosch schrieb 2002 [21]:

„Man kann davon ausgehen, dass die verzögert einsetzende Diagnose und Therapie noch entscheidender sind als die Art des therapeutischen Vorgehens in der weiteren Prognose des Ellenbogengelenks.“

Die Diagnostik des Ellenbogeninfekts setzt sich, analog zu anderen Gelenkinfektionen, zusammen aus:

\section{Anamnese}

Erhebung der ellenbogenassoziierten Beschwerden (Beginn, Dauer, Rhythmik etc.) und spezifischer Kofaktoren wie [34,35]:

- rheumatoide Arthritis

- einliegende Prothese
- intravenöser Drogenabusus

- Alkoholismus

- Diabetes

- stattgehabte intraartikuläre (Kortison) Injektionen

Wesentlich ist bei der Anamnese insbesondere die Frage nach Beschwerden an anderen Lokalisationen, da in bis zu 25\% der Fälle ein multiartikulärer Befall vorliegt [7]. Besonderes Augenmerk gilt dabei der Hüfte und dem Knie [34]. Die Beschwerdedauer wird bei Erstvorstellung der Patienten beim akuten Infekt häufig mit 1-2 Wochen angegeben [35].

\section{Klinische Untersuchung}

Gelenkinfektionen sind in ihren klinischen Escheinungsformen vielfältig und können diagnostische und differenzialdiagnostische Schwierigkeiten bereiten [47]. Da Infektionen des Ellenbogens an sich selten sind, ergeben sich daraus eo ipso z. T. erhebliche Probleme bei der Erstellung der korrekten Diagnose.

Neben den klassischen Infektzeichen sollte folgenden Faktoren besondere Aufmerksamkeit gezollt werden [26]:

- der gezielten Suche nach Narben (Inzisionen), Haut - Muskellappen - Plastiken, Verhärtung der Weichteile (Fibrose)

- dem Ausmaß der Gelenkbeweglichkeit in allen Bewegungsebenen

- der Art einer potenziellen Bewegungseinschränkung (,harter“/,weicher“ Anschlag, schmerzhafte/schmerzfreie Bewegung)

- Krepitationen

- der Gelenkachse (Valgus-Varus-Stellung des Gelenks)

Weiterhin empfehlen Kokkaklis et al. eine neurologische Untersuchung zum Ausschluss von Läsionen besonders des N. ulnaris oder des N. interosseus posterior im Rahmen einer Infektion des Ellenbogens [26]. Im Zweifelsfall sollten elektrophysiologische Untersuchungen durchgeführt werden, um das Ausmaß der Nervenschädigung zu detektieren.

Die typischen Entzündungszeichen sind auch beim Ellenbogeninfekt (außer beim hochakuten Infekt) oftmals larviert und nicht immer sind sie alle nachweisbar. Hinzu kommt, dass die Symptome nicht pathognomonisch für die infektiöse $\mathrm{Ar}$ thritis sind und ebenso bei anderen, nicht erregerinduzierten Arthritiden zu finden sind. Etwas eindeutiger ist die Sachlage beim postoperativen Gelenk- infekt. Hier sind regelhaft Zeichen der Entzündung an den operativen Zugängen zu finden, oftmals besteht eine Sekretion. Fieber ist häufig, aber nicht immer anzutreffen [46].

\section{Bedeutung der supratrochlearen Lymphknoten}

Die Nn. lymphatici supratrochleares finden sich ca. $2 \mathrm{~cm}$ proximal des Ellenbogens medialseitig am Oberarm im Septum intermusculare zwischen $M$. biceps und M. triceps brachii. Ihre Zahl variiert. Ihre Aufgabe ist die lymphatische Dränage des Ellenbogens. Im Falle der septischen Arthritis des Ellenbogens sind die supratrochlearen Lymphknoten vergrößert, entzündet und äußerst empfindlich [40].

\section{Gelenkpunktion}

Sie wird bei klinischem V.a. eine Ellenbogeninfektion in der Literatur empfohlen $[21,26]$. Sie hat imperativ unter sterilen Kautelen $\mathrm{zu}$ erfolgen. Die Punktion kann von lateral-dorsal des Radiusköpfchens („soft spot“) oder von posterior in der Fossa olecrani erfolgen [21]. Die Vorbereitung des Gelenks und des Arztes zur Punktion entspricht der einer Operation (ausreichende Händedesinfektion, Maske, Kopfbedeckung, steriler Kittel und sterile Handschuhe, OP-Feld-Desinfektion, steriles Abdecken). Wesentlich ist es, der Tatsache Rechnung zu zollen, dass auch bei einem sicher diagnostizierten Gelenkinfekt im Punktat nicht in jedem Fall ein Keimnachweis zu führen ist [34].

\section{Differenzialdiagnostik der Synovialflüssigkeit}

Der Stellenwert dieser Untersuchungen ist umstritten. Insbesondere bei der Quantifizierung der Leukozytenzahl in der Synovialflüssigkeit in Bezug auf den Nachweis eines Infekts besteht in der Literatur Uneinigkeit $[1,31,32,36]$. Jerosch hält die Punktion des Gelenks bei der Unterscheidung eines Reizzustands von einem manifesten Infekt für hilfreich. Insbesondere die Zellzahl hat dabei einen hohen diagnostischen Wert (Tab. 2) [21]. Schmidt et al. halten eine Leukozytenzahl von $>25000$ für beweisend [45].

\section{Analyse der laborchemischen Parameter}

Sie orientiert sich an den bei der Behandlung der übrigen muskuloskeletalen Infektionen üblichen Usancen. Un- 
Tab. 2 Differenzialdiagnostische Überlegungen zur Abgrenzung eines Reizzustands von einem Gelenkinfekt [21].

\begin{tabular}{ll} 
Reizzustand & Infektion \\
\hline $\begin{array}{l}\text { frühes Auftreten der Symptome innerhalb } \\
\text { der ersten } 12 \mathrm{~h} \text { nach einem Eingriff }\end{array}$ & $\begin{array}{l}\text { Auftreten/Verstärkung der Beschwerden } \\
12 \mathrm{~h}-5 \mathrm{~d} \text { nach einem Eingriff }\end{array}$ \\
\hline normale Körpertemperatur & Fieber (nicht obligat) \\
\hline keine/geringe BSG-/CRP-Erhöhung & deutliche BSG-/CRP-Erhöhung \\
\hline Leukozyten im Punktat <25000 $\mu \mathrm{l}$ & Leukozyten im Punktat $>35000 \mu \mathrm{l}$ \\
\hline & stärkeres Krankheitsgefühl, verstärkter \\
& Nachtschmerz
\end{tabular}
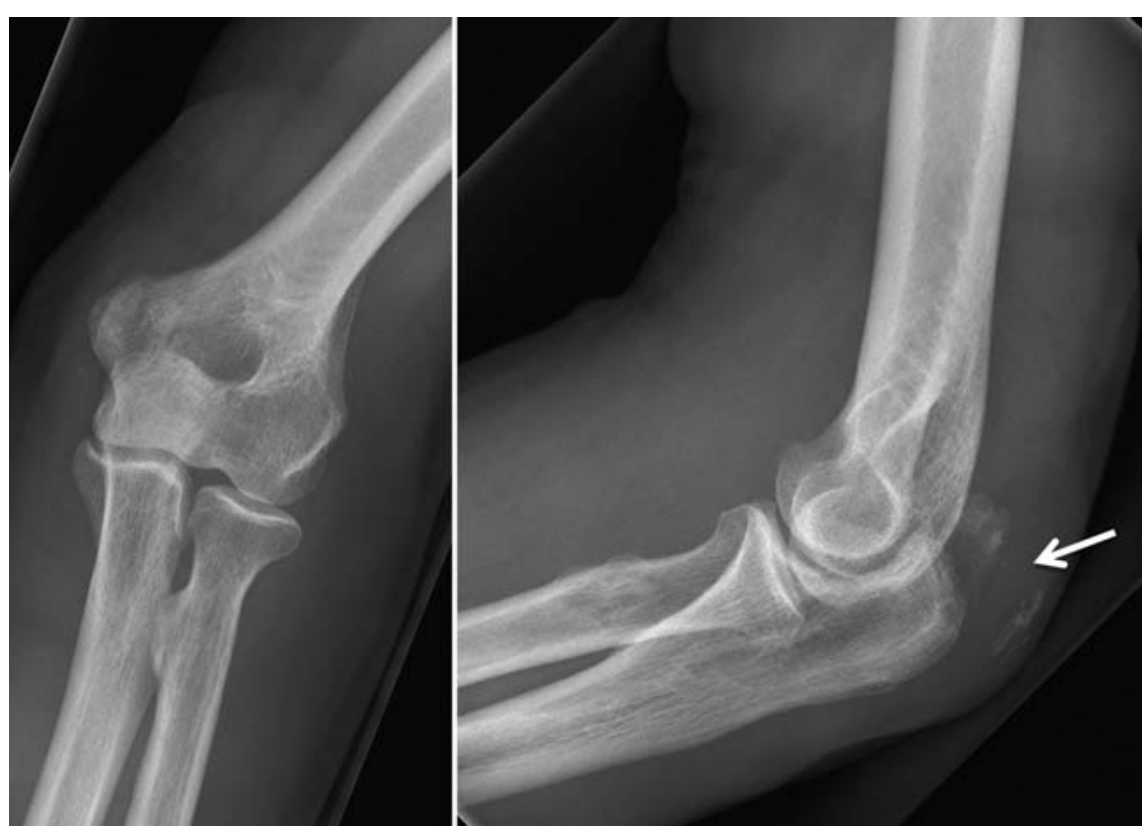

Abb. 1 Beispiel radiologische Diagnostik - Projektionsradiografie Ellenbogen in 2 Ebenen - entzündungsbedingte Verkalkungen (Pfeilkopf).

verändert spielen die Leukozytenzahl und der CRP-Wert (C-reaktives Protein) die zentrale Rolle. Dabei ist zwischen 2 Qualitäten zu unterscheiden:

- dem absoluten Wert beider Parameter an sich

- dem Werteverlauf im Rahmen der Therapie

Die absoluten Werte der klassischen Entzündungsparameter sind ein Hinweis für die Akuität der Erkrankung, der Verlauf zeigt die Wirkung der Therapie. So sollten die Werte sich bspw. postoperativ sukzessive normalisieren.

\section{Bildgebende Diagnostik}

Ausgangspunkt der bildgebenden Diagnostik bei Erst- und Verlaufsbehandlung von Gelenkinfekten ist die Projektionsradiografie in 2 Ebenen. Herauszustellen ist da die digitale Dokumentationstech-
Ist das „Röntgen“ bei bestehendem klinischen Verdacht nicht zielführend, schließt sich die Kernspintomografie als elektive Nachweis- oder Ausschlussmethodik an. Im Hinblick auf die hervorragende Darstellung von Flüssigkeitsbefunden und Weichteilinfiltraten kann sowohl intraartikulär als auch extraartikulär ein Entzündungsbefund hoch wahrscheinlich gemacht werden (Abb. 2).

Schwierigkeiten ergeben sich evtl. bei der Schweregradbeurteilung. Intraossäre Mitbeteiligungen (Knochenmarködematisierung) sollten nicht überbewertet werden (Abb.3b). Erst kontrastmittelgestützte Enhancement-Befunde können als Beweis der intraossären Abszedierung gewerten werden (Abb. $\mathbf{2}$ b und $\mathbf{3} \mathbf{a}$ ).

Besondere Aufmerksamkeit ist auf eine ggf. synoviale Reaktion bei Low-GradeBefunden zu legen. Im Hinblick auf die prognostische Bewertung von Frühinfekten sollte in den ersten 6 Wochen einer kernspintomografischen Untersuchung eine besondere Bedeutung zukommen.

In Anlehnung an die Synovialveränderungen endoskopischer Klassifikation nach Gächter können entsprechende Veränderungen auch (Graduierungsversatz beachten) kernspintomografisch erhoben werden.

Unter operationstaktischen Überlegungen wird sich regelhaft jedoch die Computertomografie anschließen. Vorteil des Schnittbildverfahrens ist die überlagerungsfreie Darstellung der ossären und Weichteilkompartimente sowie deren ggf. entzündliche Mitbeteiligung. Intraartikuläre Flüssigkeitskollektionen bzw. deren Durchbruch in die Weichteile können kompartmentbezogen validiert werden (Abb. 4).

Im Hinblick auf die klinische und prognostische Bedeutung von Gelenkentzündungen generell und den nur bedingt zugänglichen Untersuchungsregionen mittels Sonografie sollte die Projektionsradiografie als Basisuntersuchung, die Kernspintomografie als Ausschluss- oder Nachweismethode und die Computertomografie für operationstaktische Überlegungen eingesetzt werden.

\section{Mikrobiologische Analyse}

Bei allen muskuloskeletalen Infektionen spielt die mikrobiologische Untersuchung (Erregerdiagnostik) eine wesentliche Rolle und ist die Grundlage für die 


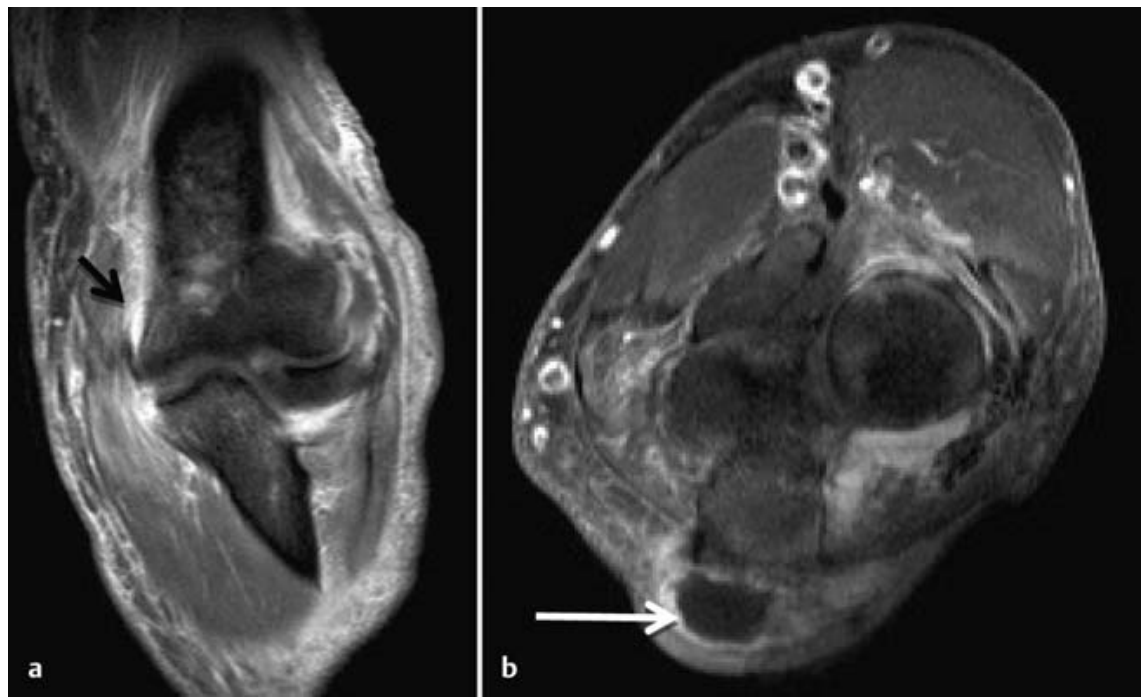

Abb. 2 a und b Beispiel radiologische Diagnostik - MR des Ellenbogens. a Koronare Schnittorientierung, b axiale Schnittorientierung. In beiden Schnittorientierungen ist gut die Weichteilentzündung (schwarzer Pfeil) und eine Abszedierung (weißer Pfeil) erkennbar.
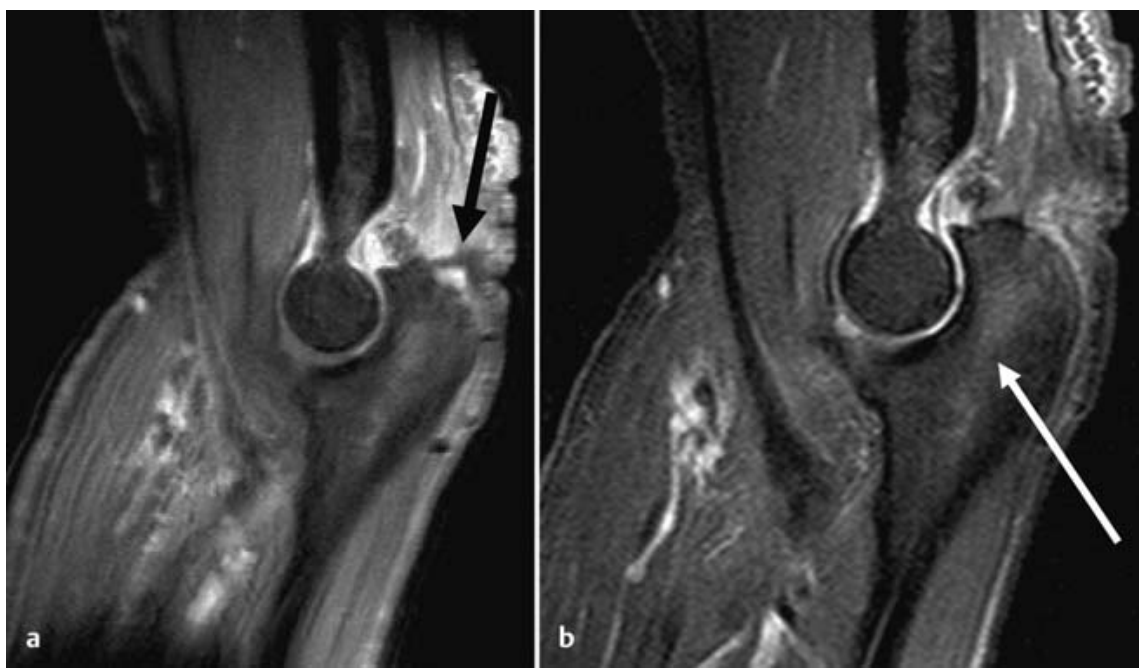

Abb. 3 a und $\mathbf{b}$ Beispiel radiologische Diagnostik-MR des Ellenbogens. Sagittale Schnittorientierung 2 Monate nach Abb. 2. Erkennbar sind eine perkutane Fistel (schwarzer Pfeil) sowie eine intraossäre Begleitentzündung (weißer Pfeil).

stadiengerechte, erregerdeterminierte Antibiotikatherapie. Nach Lew 2004 stellt sie heute in Kombination mit der ebenfalls unverzichtbaren histologischen Untersuchung den „Goldstandard“ der Diagnostik derartiger Erkrankungen dar [30]. Es ist jedoch hinlänglich bekannt, dass insbesondere die Infektionen des muskuloskeletalen Systems sich charakteristischerweise oftmals durch geringe Keimzahlen auszeichnen. Insofern kann, selbst bei Vorliegen eines ganz offensichtlichen Infekts des Ellenbogens, ein Erregernachweis nicht immer gelingen. Die Untersuchungen von Weston et al. aus dem Jahr 1999 zeigen bspw., dass die GramFärbung aspirierter Synovialflüssigkeit (intraoperativ) immer auch Gewebeproben entnommen und untersucht werden. Im Falle des Ellenbogengelenks aus allen zugänglichen Gelenkkompartimenten ebenso wie aus dem Knorpel. Abstriche aus Fistelgängen oder oberflächlichen Läsionen am Gelenk sind sinnlos, da die nachzuweisenden Erreger nicht mit den infektionsauslösenden Keimen identisch sein müssen. Geht der Infekt makroskopisch über das eigentliche Gelenk hinaus, sollte auch hier der Erregernachweis angestrebt werden, um das wahre Ausmaß der Infektion zu erkennen.

\section{b. Anzahl der Proben}

Aus den o.g. Gründen steigt mit der Anzahl entnommener Proben die Wahrscheinlichkeit, den ursächlichen Erreger zu detektieren.

\section{c. Zeitpunkt der Probenentnahme}

Wann immer möglich, sollten die Proben zu einer sicher antibiotikafreien Zeit entnommen werden, um eine Verfälschung der Ergebnisse (z.B. falsch negatives Ergebnis) zu verhindern. Der anschließend schnellstmögliche Probentransport in das Untersuchungslabor ist bei den häufig empfindlichen Erregern eine Conditio sine qua non.

Ein weiterer entscheidender Punkt bei der Bewertung der Erregerdiagnostik ist ihre Validierung im Kontext der o.g. anderen diagnostischen Parameter, wesentlich dabei ist immer die kritische Analyse der Plausibilität der Ergebnisse, speziell bei dem Verdacht auf eine Kontamination [10].

Ggf. sind die Proben einer PCR-basierten molekularen Erregerdiagnostik zuzuführen. Diese bewährt sich insbesondere für den Nachweis mykobakterieller Infektionen [28].

nur in 50\% den infektursächlichen Keim erkennen lässt. Gemeinsam mit der Kultur steigert sich dieser Wert auf 67\%. Im Umkehrschluss bleiben aber immer noch 33\% falsch negativer Ergebnisse [51].

Bei der mikrobiologischen Untersuchung spielen folgende Faktoren eine Rolle:

\section{a. Art der Probe}

Im Gegensatz zur Osteitis wird die Aspiration von Gelenkflüssigkeit im Schrifttum empfohlen [26,34,35]. Mathews empfiehlt die Punktion des geschwollenen, überwärmten Gelenks unter allen Umständen [35]. Grundsätzlich sollten

\section{Histopathologischer Infekt - Diagnostik}

Wie bereits ausgeführt gehört die histopathologische Diagnostik heute zum Goldstandard der Diagnostik [50]. Sie folgt den identischen Kautelen wie bei der mikrobiologischen Erregerdiagnostik und dient [27]:

- der Differenzierung der vorliegenden Infektsituation im Gelenk (akute/ chronische Infektion, spezifische Infektion ...)

- der Differenzierung einer Infektsituation von anderen Entitäten (z.B. Er- 


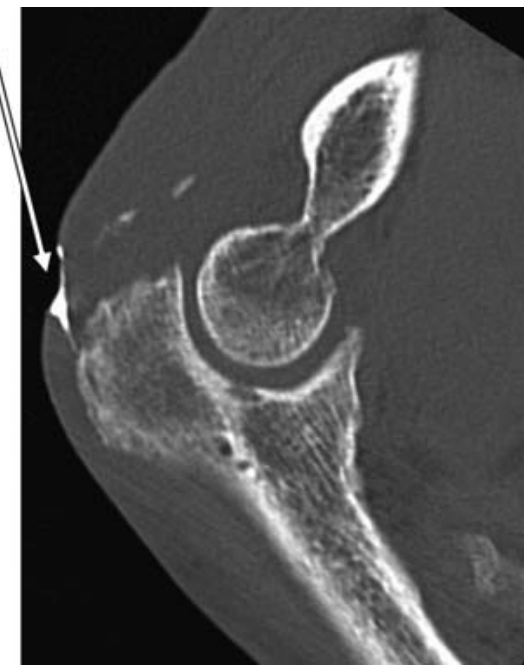

Abb. 4 Beispiel radiologische Diagnostik-CT des Ellenbogengelenks in sagittaler Schnittorientierung: 2 Monate nach Abb. 2. Erkennbar ist eine perkutane Fistel (weißer Pfeil).

krankungen aus dem rheumatoiden Formenkreis)

- dem Nachweis eines Gelenkinfekts bei negativem Erregernachweis

\section{Therapie}

In der Literatur herrscht Uneinigkeit über die Wertung und Wichtung der konservativen und operativen Maßnahmen bei der Behandlung von Gelenkinfektionen. Dieses lässt sich auch auf die Behandlung der Ellenbogengelenkinfektionen übertragen [45]. Grundsätzlich basiert die Behandlung des Gelenkinfekts auf folgenden Prinzipien $[21,49]$ :

- chirurgische Sanierung

- adjuvante Therapie (Applikation von Antibiotika)

Hierbei werden folgende Ziele verfolgt:

- Erhalt/Wiederherstellung eines funktionsfähigen belastbaren Gelenks: primäres Ziel

- Infektberuhigung und Vorbereitung eines Gelenks für eine Arthroplastik: sekundäres Ziel

- Infektberuhigung und Vorbereitung einen Gelenks für eine Arthrodese: tertiäres Ziel

\section{Konservative Behandlung (Synonym: adjuvante Therapie)}

Hierunter wird die Applikation von Antibiotika subsumiert. Auch wenn die systemische Gabe von Antibiotika bei der Behandlung von Gelenkinfektionen heute ein Standardvorgehen darstellt, so
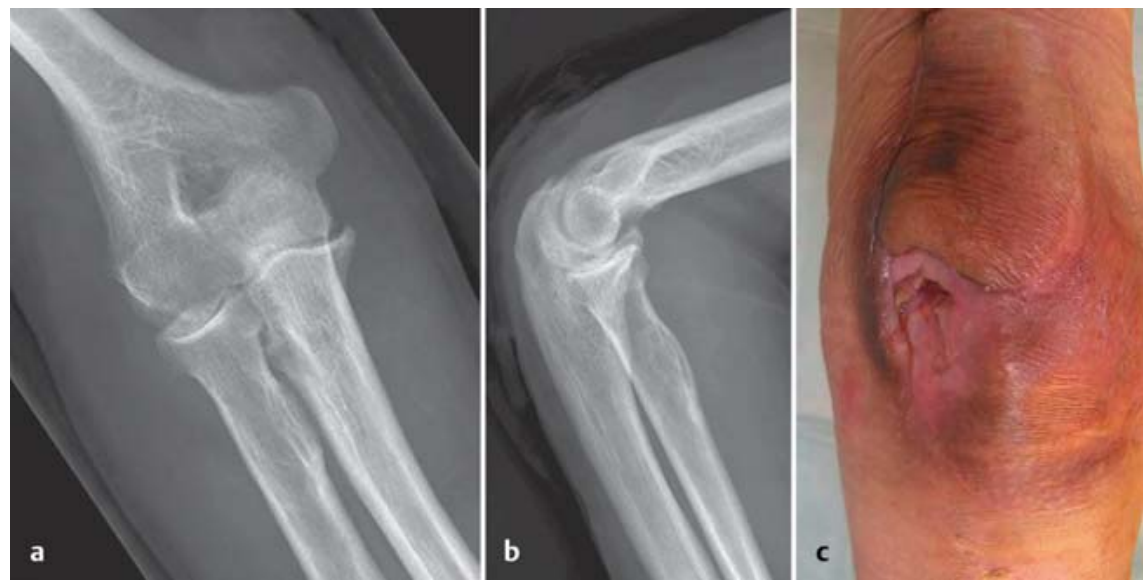

Abb. 5 a bis c 58-jähriger Mann. Diagnose: chronisch fistelnder Infekt des rechten Ellenbogengelenks. In Behandlung seit Juni 2010. Erstvorstellung: April 2011. Nebendiagnosen: insulinpflichtiger Diabetes mellitus Typ 2; COPD; arterielle Hypertonie; Vorhofflimmern bei Tachycardia absoluta; chronischer Alkoholabusus; chronisches Krampfleiden. Keim: Staphylococcus epidermidis. Labor: Leukozytenzahl 9,6 Gpt/l; CRP 69 mg/l. Voroperationen: $10 \times$ radikales Débridement des Gelenks. a Projektionsradiografie: a.-p. Bild. Kein Anhalt für ossäre Destruktion. b Projektionsradiografie: Seitliches Bild. Kein Anhalt für ossäre Destruktion. c Klinisches Bild. Gut erkennbar die chronische Fistel sowie die Narbenbildung nach mehrfacher operativer Revision.

wird die konkrete Ausführung dieser Maßnahmen in der Literatur nicht einheitlich beschrieben. Im Gegenteil. Eine Metaanalyse aus dem Jahr 2001 konnte kein Antibiotikumregime identifizieren, welches anderen bei der Behandlung von Gelenkinfektionen signifikant überlegen war [48]. Konsens besteht darüber, dass sich die Auswahl der Antibiotika im Falle der kalkulierten Therapie an den wahrscheinlichsten Erregern (i.e. S. aureus) orientieren sollte [35]. Weiterhin sollte bei der Auswahl der Antibiotika der stetig steigenden Rate von MRSA-Infekten Rechnung getragen werden.

\section{Operative Behandlung}

Die grundsätzliche Problematik der chirurgischen Revision des Ellenbogengelenks resultiert aus seinem anatomischen Aufbau. Das Ellenbogengelenk verbindet 3 Knochen in 3 Teilgelenken (Articulatio humeroulnaris, humeroradialis, radioulnaris prox.) Die Gelenkkapsel ist fest und eng und umschließt die artikulierenden Teile sowie an der Vorder- und Hinterseite des Humerus ausgeprägte Gruben. Die Epikondylen liegen extraartikulär [6].

Die generelle Frage nach der Rolle der chirurgischen Intervention bei der septischen Arthritis wird im Schrifttum durchaus kontrovers diskutiert [33]. So verglichen Ravindran et al. 2009 die nichtoperative (medikamentöse) Therapie mit der operativen (Arthroskopie/Arthrotomie). In ihrer Untersuchung konn- ten die Autoren keinen eindeutigen Vorteil in einem der beiden therapeutischen Ansätze erkennen [41]. Nach Mathews et al. stellt die Entfernung der purulenten Flüssigkeit aus dem infizierten Gelenk einen wesentlichen Bestandteil der Behandlung dar [34].

Die Vorgehensweise bei der operativen Gelenkrevision lässt sich, analog zu anderen Gelenken aus dem nach Schmidt modifizierten Gächter-Schema ableiten [46]. Hierbei orientiert sich die Indikation für ein arthroskopisches oder ein offenes Vorgehen an (Tab. 1):

- dem Gächter-Stadium der Erkrankung

- der Vorbehandlung

- der Infektausdehnung

Neben der arthroskopischen und der offenen Gelenkrevision (s.u.) bietet sich dafür grundsätzlich auch die Nadelaspiration an [34]. In einer Metaanalyse aus dem Jahr 2007 konnten Mathews et al. interessanterweise die Frage nach der zu bevorzugenden Vorgehensweise nicht eindeutig beantworten [33].

Arthroskopische Operationen am Ellenbogen stehen in der Häufigkeit weit hinter vergleichbaren Eingriffen am Kniebzw. Schultergelenk. Entsprechend geringer sind die jährlichen Operationszahlen pro Operateur. Spezifische anatomische Besonderheiten und damit verbundene Gefahrenmöglichkeiten erfordern gute Kenntnisse und Fertigkeiten hinsichtlich der operativen Ausführung [47,52]. 
Die Besonderheit der Arthroskopie des Ellenbogens gründet sich [4]:

- auf die komplexe anatomische Struktur des Gelenks

- die enge anatomische Nachbarschaft zu neurovaskulären Strukturen (s.u.)

- das geringe Kapselvolumen

Insofern relativieren sich die grundsätzlichen Vorteile der arthroskopischen Gelenkrevision im Falle des Ellenbogens [49]:

- minimalinvasive und somit minimal traumatisierende Technik

- sehr gute Darstellbarkeit der anatomischen Strukturen

- schnellere Rekonvaleszenz nach dem Eingriff

- bessere funktionelle Ergebnisse

Grundsätzlich sind die septischen $\mathrm{Ar}$ thritiden des Ellenbogengelenks der Arthroskopie zugänglich. Nach Jerosch eignen sich besonders akute Infektionen nach Gelenkinjektionen oder -instillationen für dieses Vorgehen [20]. Im Frühstadium dieser Erkrankungen, nach Schmidt et al. innerhalb der ersten 7 Tage, kann die konsequente arthroskopische Spülung den gelenkdestruierenden Prozess möglicherweise stoppen $[20,45]$. Im Falle von stattgehabten Voroperationen muss individuell entschieden werden, ob ein ausschließlich arthroskopisches Vorgehen ausreichend ist oder eine (semi-)offene Technik die zielführende Variante darstellt [52]. Im eigenen Vorgehen werden hierzu die o.g. Kriterien der nach Schmidt modifizierten Gächter-Klassifikation herangezogen. Die arthroskopische Synovektomie des Ellenbogens wurde von Lee und Morrey 1997 untersucht und beschrieben. Diese Autoren zeigten auch den wesentlichen Nachteil der Methode auf, nämlich die Tatsache, dass eine komplette Synovektomie des Ellenbogengelenks arthroskopisch nicht möglich ist [29]. Dieses deckt sich mit der grundsätzlichen Annahme, dass die arthroskopische Gelenkrevision immer dann an ihre Grenzen stößt, wenn [49]:

- der Infekt das eigentliche Gelenk überschreitet,

- der Infekt sich in arthroskopisch schlecht oder nicht erreichbaren Gelenkabschnitten befindet.

Eine Reihe von Autoren verwiesen nachdrücklich auf das Risiko der neurovaskulären Komplikationen bei der arthroskopischen Synovektomie des Ellenbogens [25].

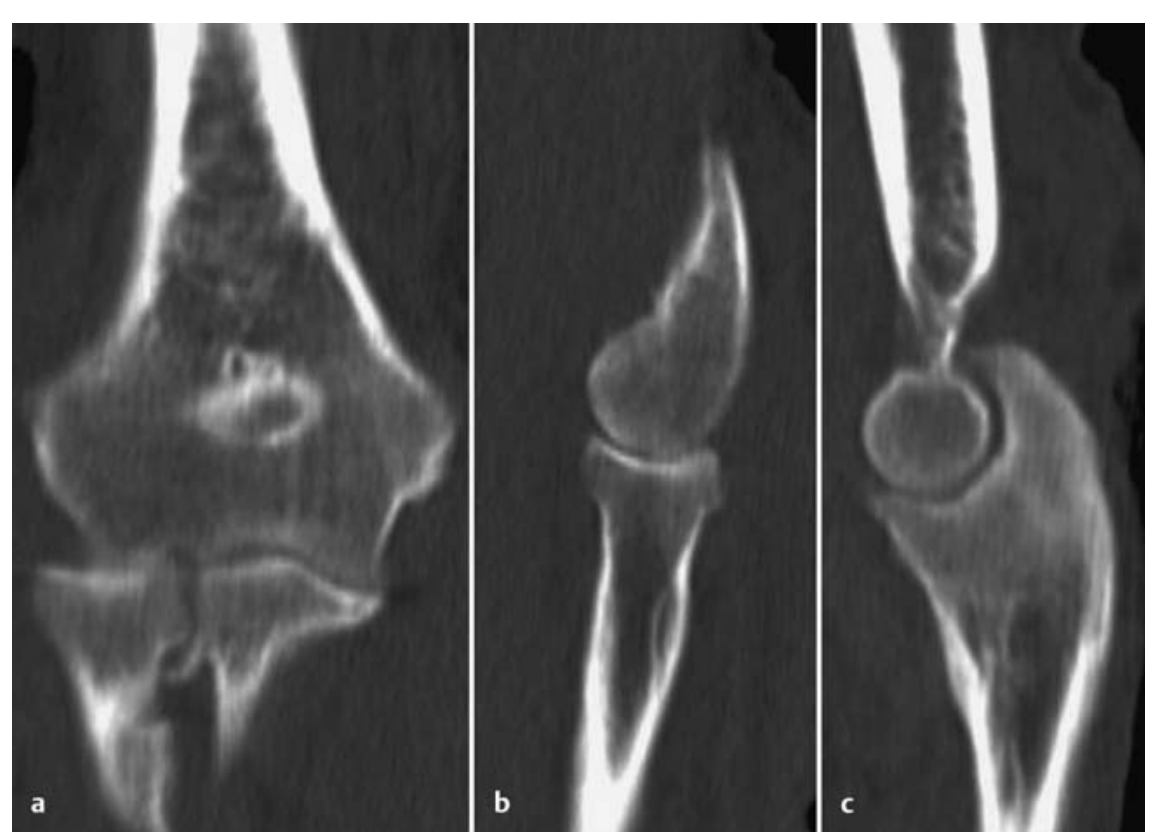

Abb. 6a bis c CT vom 19.04.2011: kein Anhalt für signifikante, infektinduzierte Knochendestruktion. Jedoch gut erkennbare Verschmälerung des Gelenkspalts in allen Kompartimenten. a A.-p. Bild, b seitliches Bild humeroradial, c seitliches Bild humeroulnar.
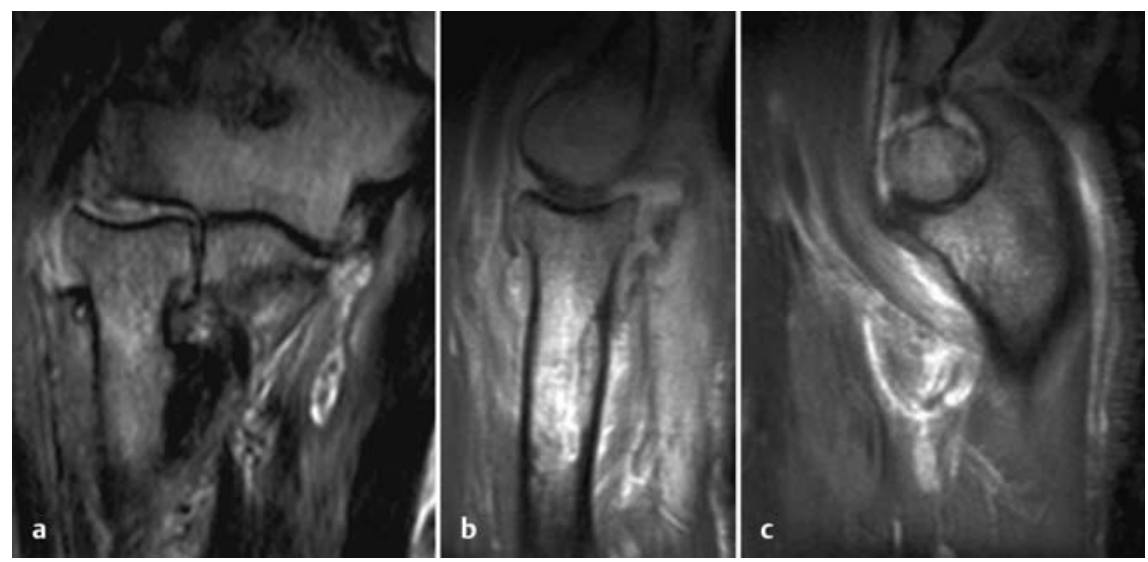

Abb.7a bis c MRT vom 19.04.2011: gut erkennbar die entzündlichen Veränderungen im Bereich der Weichteile sowie des Knochens (diffuse entzündliche Infiltrationen am Mm. supinator, brachialis, anconaeus. Begleitosteitis am distalen Humerus, proximalen Radius, proximaler Ulna). a A.-p. Bild, b seitliches Bild humeroradial, c seitliches Bild humeroulnar.

Die Gelenklavage geschieht sowohl beim offenen als auch beim arthroskopischen Vorgehen mit physiologischer Kochsalzlösung (im eigenen Vorgehen nicht unter 61). Für die antiseptischen Lösungen gilt das bereits bei den Knocheninfektionen Gesagte.

Die Anwendung von Saug-Spül-Drainagen gilt heute allgemein als obsolet.

Das Einbringen von Antibiotika-Vliesen in die betroffenen Gelenke wird in der Literatur unterschiedlich bewertet. Während einige Autoren dieses Vorgehen empfehlen, warnen andere vor dem
Auftreten einer chemisch induzierten Synovitis [4]. Die systemische Antibiotikatherapie gehört, wie bei den Knocheninfektionen zu den adjuvanten Maßnahmen und unterstützt das chirurgische Management.

Die postoperative Verlaufsbeobachtung stützt sich auf die engmaschige Kontrolle von Klinik und paraklinischen Parametern. Der Zeitpunkt für evtl. notwendige Revisionen wird in der Literatur unterschiedlich angegeben. Einige Autoren machen ihn abhängig von Klinik, Paraklinik und mikrobiologischem Ergebnis [17]. In Abhängigkeit von der Akuität 


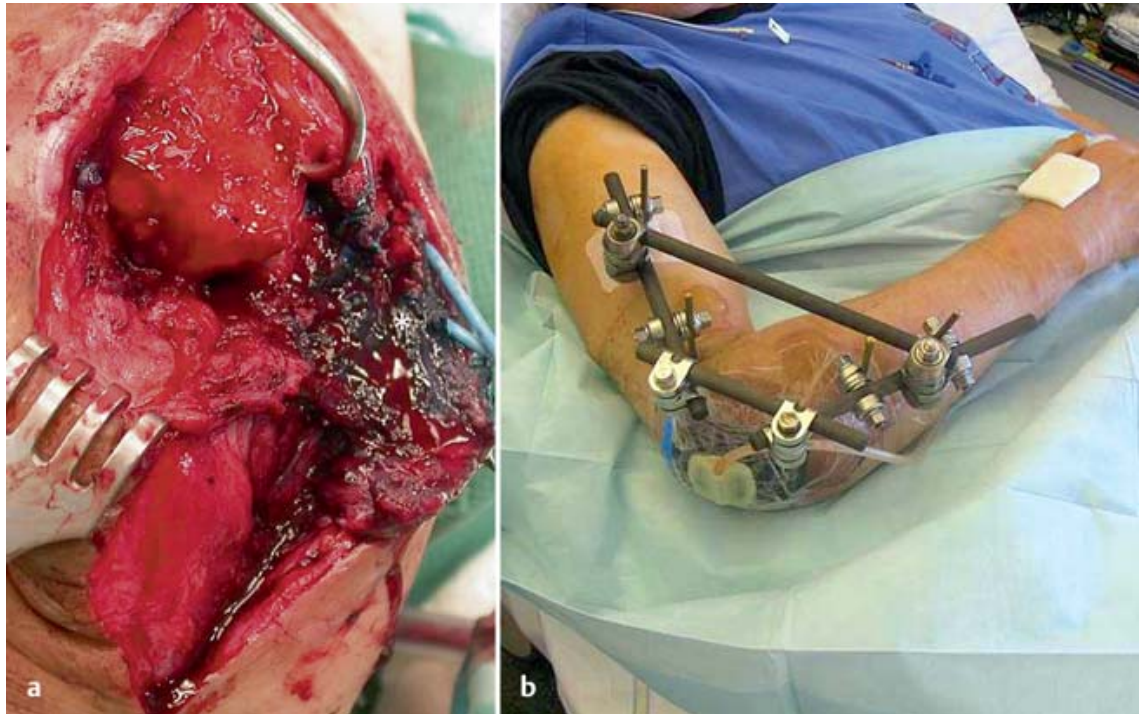

Abb. 8a und b Aufgrund der chronischen Infektsituation nach intensiver Aufklärung des Patienten Entschluss zur Ellenbogengelenkresektion. a Intraoperativer Befund vom 27.04.2011 nach Eröffnung des Gelenks. Unter dem Einzinker befindet sich das Olecranon ulnae. Der N. ulnaris ist mit einem blauen Vessel Loop angeschlungen. Gut zu erkennen ist das sulzige Gewebe $\left.{ }^{*}\right)$, welches für einen chronischen Gelenkinfekt typisch ist. Bei den insges. bereits präoperativ prekären Weichteilen wurde die Gelenkrevision über den ursprünglichen dorsalen Zugang durchgeführt. b Postoperativer Befund. Wegen der Instabilität des „Gelenks“ und zur Weichteilprotektion ist ein Fixateur externe angelegt worden. Der verbliebene Weichteildefekt wurde mittels eines Vakuumverbands verschlossen.

der Erkrankung führen wir im eigenen Vorgehen eine Stufentherapie durch, die sich an folgenden Parametern orientiert:

- am Gächter/Schmidt-Stadium der Erkrankung $[11,12,19,46]$.

- der Klinik

- dem Verlauf der paraklinischen Infektparameter

\section{Nachbehandlung und Rehabilitation}

Das wesentliche Problem scheint à la longue weniger die Gefahr des Infektrezidivs oder der Infektpersistenz zu sein als der Funktionsverlust [22]. Insofern hat die frühfunktionelle Behandlung unter ausreichender Analgetikagabe einen sehr hohen Stellenwert. Sie inkludiert physiotherapeutische Maßnahmen mit aktiver und passiver Bewegung des betroffenen Gelenks ebenso wie den Einsatz von Bewegungsschienen. Nur dadurch kann die Funktion des Gelenks erhalten werden. Auch Schmidt et al. empfahlen dieses Vorgehen 2000 [44]. Im eigenen Vorgehen beginnt die funktionelle Behandlung am Tag nach der Operation. Die postoperative Aufbelastung des Gelenks empfiehlt Jerosch nicht vor der 6. postoperativen Woche [21].

\section{Postinfektiöse Folgezustände}

Nicht in allen Fällen lässt sich mit den o.g. Maßnahmen das Ellenbogengelenk erhalten. In besonders komplexen Fällen mit über das eigentliche Gelenk hinausgehendem Infekt ist die Resektion des Gelenks notwendig. In derartigen Fällen kommen im Wesentlichen 3 Möglichkeiten zur weiteren Behandlung in Betracht:

Die endoprothetische Versorgung. Sie stellt immer dann eine gute Option dar, wenn:

- der Infekt beruhigt ist,

- der Betroffene keiner schweren körperlichen Arbeit nachgeht, die zu einer Überbelastung der Prothese und damit zu ihrem Versagen führen kann (älterer Patient).
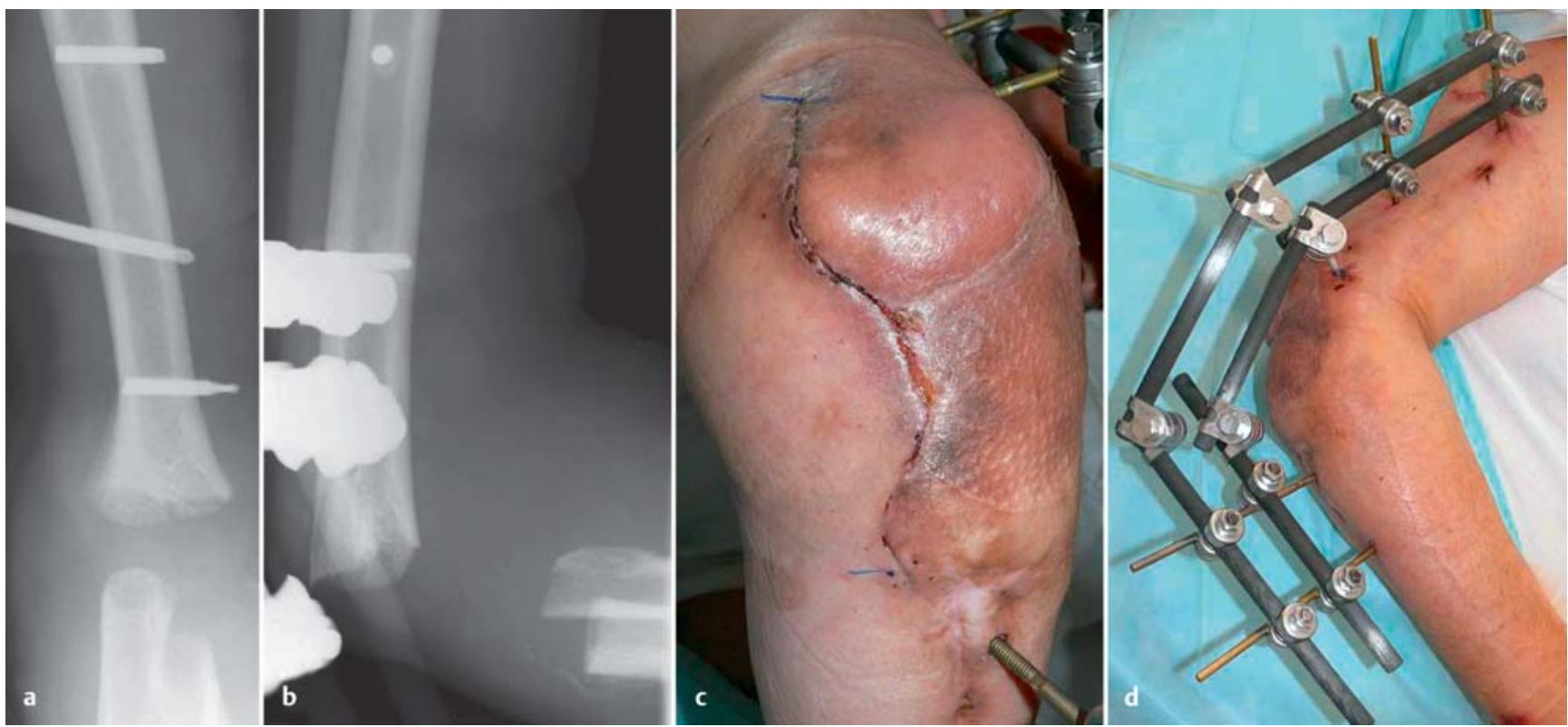

Abb. 9 a bis d Zustand vom 29.06. 2011. Nach erneuter Wundrevision und Fixateurumbau (Pinbruch nach Krampfanfall). a Projektionsradiografie: a.-p. Projektion. b Projektionsradiografie: seitliches Bild. c Reizlose Wundverhältnisse. Intrakutane Hautnaht zur Entlastung der Wundränder. d Im Fixateur externe fixiertes Ellenbogengelenk. 


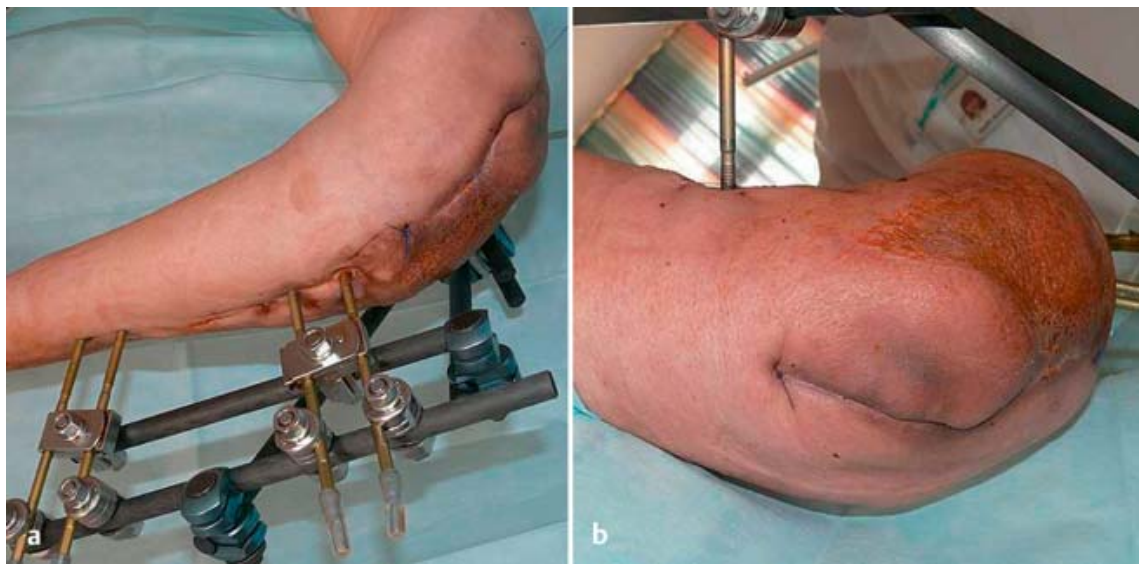

Abb. 10a und b Klinischer Befund vom 28.07.2011. Reizlose Weichteilverhältnisse. Kein Anhalt für floriden Infekt.
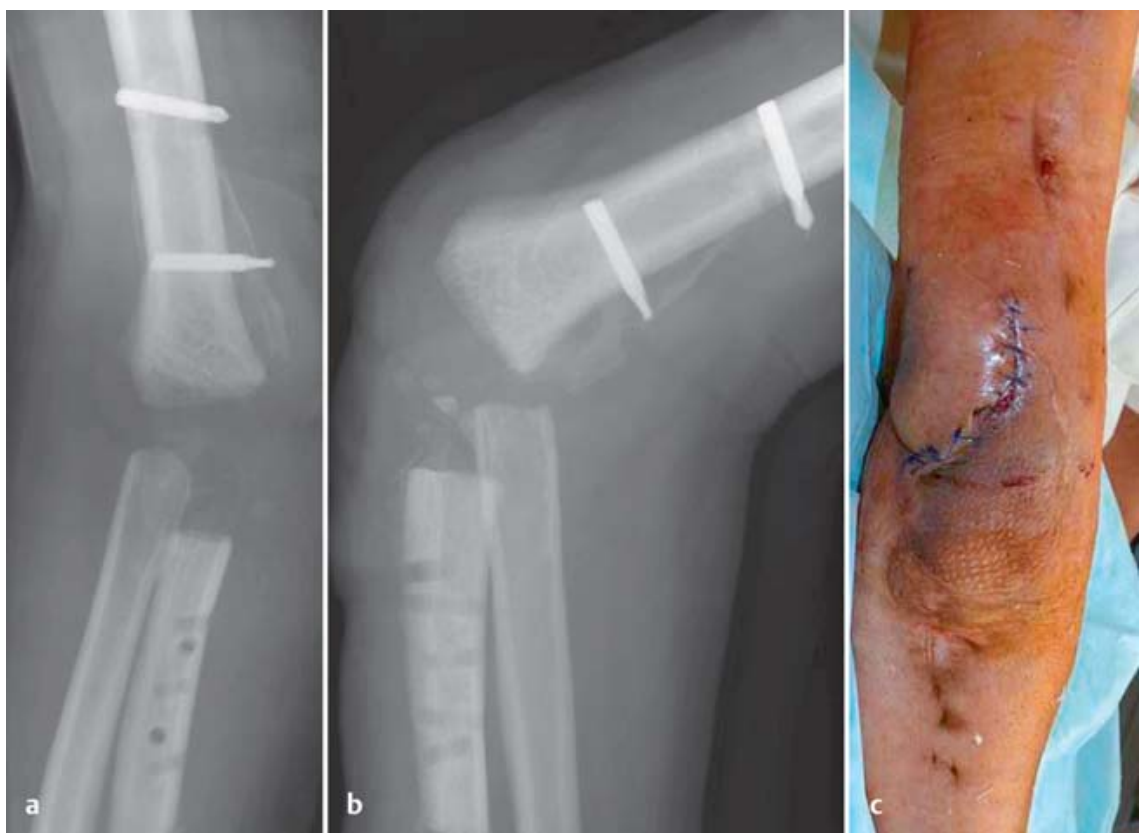

Abb. 11 a bis c Radiologisches und klinisches Bild bei der letztmaligen Wiedervorstellung am 19. 12. 2011. a A.-p. Bild. b Seitliches Bild. c Klinisches Bild. Wunde reizlos. Kein Anhalt für floriden Infekt. Keine Sekretion. Stabilisierung des Gelenks mittels Hülsenapparat.

Die Ellenbogengelenkarthrodese. Gerade nach Infektionen und schmerzhaften Bewegungseinschränkungen kann sie eine Schmerzreduktion bei gleichzeitiger guter Kraftentfaltung herbeiführen [38]. Hiervon profitieren jüngere, körperlich höher belastete Patienten, die hohe Anforderungen an die Funktion der oberen Extremität stellen [24]. Kälicke empfiehlt im Falle des Ellenbogeninfekts ein 2-zeitiges Vorgehen [24]:

Schritt 1: Resektion des infizierten Gelenks und Stabilisierung des Ellenbogens mittels Fixateur externe

Schritt 2: Arthrodese mittels interner Osteosynthese
Die Resektionsarthroplastik des Ellenbogens. Sie ist die Ultima Ratio bei therapierefraktärer Infektion [53]. In der Literatur findet dieses Vorgehen in den letzten Jahren nur wenig Erwähnung. Im deutschen und italienischen Schrifttum wird sie in den 80er- und 90er-Jahren erwähnt (Abb. 5-11) [16,42].

In der Literatur sind weitere Verfahren zur Rettung des Ellenbogens beschrieben, die aber sicherlich nur in Ausnahmesituationen in der Infektsituation zu Anwendung kommen. Beispielhaft sei die Interpositionsarthroplastik genannt.

Im Vergleich zur oben erwähnten Resektionsarthroplastik werden die destruier- ten Gelenkanteile möglichst sparsam reseziert und so ein annähernd kongruentes Humeroulnargelenk erhalten. Heutzutage werden nach Resektion der Gelenkflächen Fascia-lata-Transplantate oder alternativ allogene Präparate der Achillessehne aufgelegt [24]. Nach Kälicke ist die Indikation zur Interpositionsarthroplastik immer dann gegeben, wenn arthrotische Veränderungen des Gelenks vorliegen, eine endoprothetische Versorgung jedoch nicht in Betracht kommt [24]. Postinfektiöse Zustände gehören demnach nicht zu den klassischen Indikationen.

Sicherlich darf auch der Umstand nicht vergessen werden, dass im Falle des ausgedehnten Infekts mit gleichzeitiger vitaler Bedrohung des Patienten die Indikation Amputation gegeben sein kann („life before limb“).

\section{Fazit}

- Infektionen des Ellenbogengelenks bedürfen einer konsequenten und zügigen Diagnostik und Therapie.

- Der akute Ellenbogengelenkinfekt ist ein Notfall.

- Die Symptomatik ist oftmals larviert. Wesentlich ist es, an diese Entität überhaupt zu denken.

- Die Therapie setzt sich aus chirurgischen und adjuvanten Maßnahmen zusammen.

- Die Behandlung muss stadiengerecht erfolgen.

- Die frühestmögliche postoperative Physiotherapie ist eine Conditio sine qua non.

\section{Literatur}

${ }^{1}$ Abdullah S, Young-Min SA, Hudson SJ et al. Gross synovial fluid analysis in the differential diagnosis of joint effusion. J Clin Pathol 2007; 60: 1144-1147

2 Argen RJ, Wilson CH, Wood P. Suppurative arthritis. Arch Intern Med 1966; 117: 661-664

${ }^{3}$ Arnold SR, Elias D, Buckingham SC et al. Changing patterns of acute hematogenous osteomyelitis and septic arthritis: emergence of community-associated methicillin-resistant Staphylococcus aureus. J Ped Orthop 2006; 26: 703-708

${ }^{4}$ Attmannspacher W. Arthroskopie. In Josten C, Lill H, Hrsg. Ellenbogenverletzungen. Darmstadt: Steinkopf; 2002

${ }^{5}$ Battmann A, Jürgens I. Pathophysiologie. In: Hendrich C, Frommelt L, Eulert J, Hrsg. Septische Knochen- und Gelenkchirurgie. Berlin, Heidelberg: Springer; 2004

${ }^{6}$ Bertolini R, Leutert G, Rother P et al. Systemische Anatomie des Menschen. Berlin: Verl. Volk und Gesundheit; 1988

7 Dubost IJ. Fis I, Denis P et al. Polyarticular septic arthritis. Medicine (Baltimore) 1993; 72 : 296-301 
${ }^{8}$ Dubost JJ, Soubrier M, De Champs et al. No changes in the distribution of organisms responsible for septic arthritis over a 20 year period. Ann Rheum Dis 2002; 61: 267-269

9 Frazee BW, Fee C, Lambert L. How common is MRSA in adult septic arthritis? Ann Emerg Med 2009; 54: 695-700

${ }^{10}$ Frommelt L. Antibiotikatherapie. In: Schnettler R., Steinau HU, Hrsg. Septische Knochenchirurgie. Stuttgart, New York: Thieme; 2004

11 Gächter A. Die Bedeutung der Arthroskopie beim Pyarthros. Hefte Unfallheilkd 1988; 200: 132-136

12 Gächter A. Gelenkinfekt - Arthroskopische Spülungsbehandlung - Hints und Tricks. Arthroskopie 1994; 7: 98-101

${ }^{13}$ Geirsson AJ, Stakevicius S, Vikingsson A. Septic arthritis in Iceland 1990-2002: increasing incidence due to iatrogenic infections. Ann Rheum Dis 2008; 67: 638-643

14 Goldberg DL, Brand KD, Cohen AS et al. Treatment of septic arthritis. Arth Rheum 1975; 18: 83-90

15 Gupta MN, Sturrock RD, Field M. Prospective comparative study of patients with culture proven and high suspicion of adult onset septic arthritis. Ann Rheum Dis 2003; 62: $327-$ 331

${ }^{16}$ Hahn MP, Ostermann PA, Richter D et al. [Elbow arthrodesis and its alternative.] Orthopäde 1996; 25: 112-120

17 Hendrich C, Frommelt L, Eulert J. Septische Knochen- und Gelenkchirurgie. Berlin, Heidelberg, New York: Springer; 2004

18 Hofmann GO. Infektionen der Knochen und Gelenke. München, Jena: Urban und Fischer; 2004

19 Jensen KU, Klein W, Dann K. Die arthroskopische Behandlung der septischen Gonitis. Arthroskopie 1989; 2: 104-108

20 Jerosch J, Schröder M, Schneider T. Good and relative indications for elbow arthroscopy. Arch Orthop Trauma Surg 1998; 117: 246249

${ }^{21}$ Jerosch J. Infektionen. In Josten C, Lill H, Hrsg. Ellenbogenverletzungen. Darmstadt: Steinkopf; 2002

${ }^{22}$ Josten C, Lill H, Hrsg. Ellenbogenverletzungen. Darmstadt; Steinkopf 2002

${ }^{23}$ Kaandorp CJ, Dinant HJ, van de Laar et al. Incidence and sources of native and prosthetic joint infection: a community based prospective survey. Ann Rheum Dis 1997; 56: 470-475

${ }^{24}$ Kälicke T, Weber O, Backhaus M et al. Salvage Procedures am Ellenbogen. Unfallchirurg 2010; 113: 990-995

${ }^{25}$ Kang HJ, Park MJ, Ahn JH et al. Arthroscopic synovectomy for the rheumatoid elbow. Arthroscopy 2010; 26: 1195-1202

${ }^{26}$ Kokkalis ZT, Schmidt CC, Sotereanos DG. Elbow arthritis: current concepts. JHS 2009; 34: 761-768

${ }^{27}$ Krenn V. Editorial: Orthopädische Pathologie. Der Pathologe 2011; 32: 181
${ }^{28}$ Kriegsmann J, Hopf T, Jacobs D et al. Anwendungsmöglichkeiten der Molekularpathologie bei Gelenksinfektionen. Orthopäde 2009; 38: $531-538$

${ }^{29}$ Lee BP, Morrey BF. Arthroscopic synovectomy of the elbow for rheumatoid arthritis. A prospective study. J Bone Joint Surg [Br] 1997; 79: 770-772

${ }^{30}$ Lew DP, Waldvogel FA. Osteomyelitis. Lancet 2004: 364: 369-379

${ }^{31}$ Li SF, Chang C, Gharib C et al. Diagnostic utility of laboratory teste in septic arthritis. Emerg Med 2007; 24: 75-77

${ }^{32}$ Margaretten ME, Kohlwes J, Moore D et al. Does this adult patient have septic arthritis? JAMA 2007; 297: 1478-1488

${ }^{33}$ Mathews CJ, Kingsley G, Field M et al. Management of septic arthritis: a systematic review. Ann Rheum Dis 2007; 66: 440-445

${ }^{34}$ Mathews CJ, Coakley G. Septic arthritis: current diagnostic and therapeutic algorithm. Curr Opin Rheumatol 2008; 20: 457-462

35 Mathews CJ, Weston VC, Jones A et al. Bacterial septic arthritis in adults. Lancet 2010; 375: 846-855

${ }^{36}$ McGillicuddy DC, Shah KH, Friedberg RP et al. How sensitive is the synovial fluid white blood count in diagnosing septic arthrotis? Am J Emerg Med 2007; 25: 749-752

37 Mehta P, Schnall SB, Charalampos GZ Septic arthritis of the shoulder, elbow, and wrist. Clin Orthop Relat Res 2006; 451: 42-45

38 Moghaddam-Alvandi A, Dremel E, Güven F et al. Ellenbogengelenkarthrodese. Unfallchirurg 2010; 113: 300-307

${ }^{39}$ Morgan DS, Fisher D, Merianos A et al. An 18 year clinical review of septic arthritis from tropical Australia. Epidemiol Infect 1996; 117: 423-428

40 Patel DA. The supratrochlear lymph nodes: their diagnostic significance in a swollen elbow joint. Ann R Coll Surg Engl 2001; 83: 425-426

${ }^{41}$ Ravindran V, Logan I, Bourke BE. Medical vs. surgical treatment for the native joint in septic arthritis: a 6-year, single UK academic centre experience. Rheumatology 2009; 48: 1320-1322

42 Razza F, Peretti GM, Macías G et al. Resection arthroplasty as alternative procedure for the stiff elbow. Minerva Ortop Traumatol 2001; 52: 73-80

43 Ross IJ. Septic arthritis. Infect Dis Clin North Am 2005; 19: 799-817

44 Schmidt HGK. Postoperative Infektion des Ellenbogens. Trauma Berufskrankh 2000; 2 (Suppl. 1): 86-91

45 Schmidt HGK, Gerlach U, Wurm M et al. Diagnostik und Therapie von Schulter- und Ellengelenkempyemen. Trauma Berufskrankh 2001; 3 (Suppl. 3): 404-414

46 Schmidt HGK, Gerlach UJ, Schoop R. Empyembehandlung. Trauma Berufskrankh 2005; 7 (Suppl. 1): 120-129
47 Schneider U, Hierholzer G, Böhm HJ. Knochenund Gelenkinfektionen. Unfallchirurg 1996; 99: 789-800

48 Stengl D, Bauwens K, Schouli J et al. Systematic review and meta-analysis of antibiotic therapy for bone and joint infections. Lancet Infect Dis 2001; 1 : 175-188

49 Tiemann AH. Infektiöse Erkrankungen des Bewegungsapparates. In: Krenn V, Rüther W, Hrsg. Pathologie des Bewegungsapparates. Berlin/Boston: De Gruyter; 2011

50 Tiemann AH, Hofmann GO. Knocheninfektionen. Unfallchirurg 2012; im Druck

51 Weston VC, Jones AC, Bradbury N et al. Clinical features and outcome of septic arthritis in a single UK health district 1982-1991. Ann Rheum Dis 1999; 58: 214-219

52 Witt M, Mittlmeier T. Arthroskopie des Ellenbogengelenkes. Unfallchirurg 2007; 110: 953-963

53 Zarkadas PC, Cass B, Throckmorton $T$ et al. Long-term outcome of resection arthroplasty for the failed total elbow arthroplasty. J Bone Joint Surg [Am] 2010; 92: 2576-2582

\section{Prof. Dr. med. Andreas Heinrich Tiemann}

LA der Abteilung für Septische und Rekonstruktive Chirurgie der Klinik für Unfall - und Wiederherstellungschirurgie

Dr. med. Rainer Braunschweig Direktor der Klinik für Bildgebende Diagnostik und Interventionsradiologie

BG-Kliniken Bergmannstrost Halle Merseburger Straße 165 06112 Halle

andreas.tiemann@bergmannstrost.com

\section{Prof. Dr. Dr. Gunther Olaf Hofmann}

Direktor der Klinik für Unfall- und Wiederherstellungschirurgie BG-Kliniken Bergmannstrost Halle Merseburger Straße 165

06112 Halle

Direktor der Kliniken für Unfall-, Hand- und Wiederherstellungschirurgie Friedrich-Schiller-Universität Jena Erlanger Allee 101 07747 Jena 